\title{
Radial Electron-Beam-Breakup Transit-Time Oscillator
}

Michael A. Mostrom

Mission Research Corporation, Albuquerque, New Mexico 87106

Thomas J. T. Kwan

Applied Theoretical Physics Division, Los Alamos National Laboratory, Los Alamos, New Mexico 87545

A new radially-driven electron-beam-breakup transit-time oscillator has been investigated analytically and through computer simulation as a compact low-impedance high-power microwave generator. In a $1 \mathrm{MV}, 50 \mathrm{kA}$ device $35 \mathrm{~cm}$ in radius and $15 \mathrm{~cm}$ long, with no external magnetic field, $5 \mathrm{GW}$ of extracted power and a growth rate of $0.26 / \mathrm{ns}$ have been observed. Theoretical maximum efficiencies are several times higher.

PACS numbers: $85.10 . J z, 52.35 . H r, 52.65 .+\mathrm{z}$

In previous work, we developed a new microwave generator: the Axially Driven Electron-Beam-Breakup Transit-Time Oscillator (ABTO). ${ }^{1}$ In spite of several advantages over other microwave devices, the $\mathrm{ABTO}$ is constrained by a beam impedance no lower than about $400 \Omega$. Therefore, to get high power, high voltage is required; which eliminates many possible applications. In order to increase power without going to impractically-high voltages and bulky insulation, the $\mathrm{ABTO}$ concept was radically modified to use a radial electron beam so more current could cross the cavity. This new device, the RBTO, is shown in Fig.1 for a radially inward-directed beam. The beam is generated in a coaxial vacuum transmission line, with symmetric power feed to a radial field-emission diode, and enters the cavity through a screen (indicated by the dashed line in Fig.1). It is then axially deflected like a rippled drum membrane by the cavity wave.

We have calculated the growth rate, nonlinear saturation level, and space-charge limited current of a radial beam in an annular cavity. Simulations show reasonable agreement with the growth rate predictions, and show extracted output power of $5 \mathrm{GW}$, or about $10 \%$ extraction efficiency. The saturation model predicts possible extraction efficiencies several times larger (up to $50 \%$ ).

The RBTO has several advantages: (1) the 2D TEM microwave mode is easy to analyze and compute; (2) the radial beam can carry 50 times more current and power than an axial beam ( $<10 \Omega$ impedance); (3) the high efficiency and growth rate decrease slowly with increasing voltage; and (4) the device is compact with no external magnetic field. Advantages (3) and (4) are shared with the ABTO over many other devices.

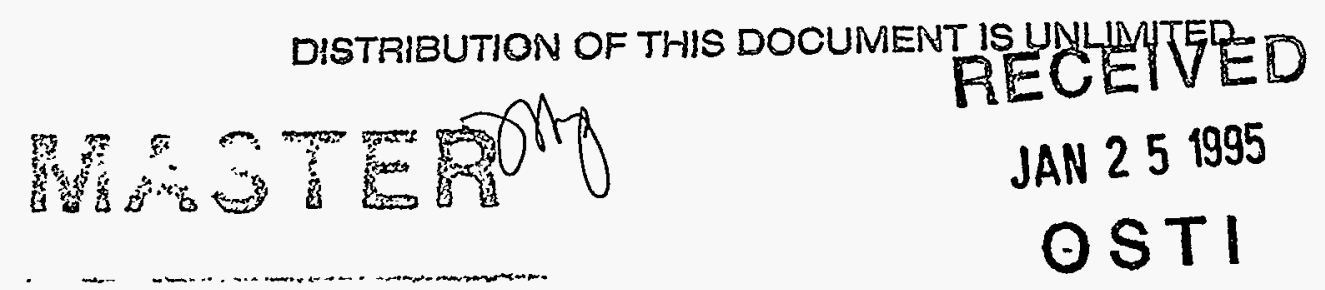




\section{DISCLAIMER}

Portions of this document may be illegible in electronic image products. Images are produced from the best available original document. 
Applications of high-power microwaves include high-average-current high-gradient accelerators, fusion plasma heating, materials processing, radar, and defense.

The ABTO device depends on the interaction of an axial beam with a $\mathrm{TM}_{110}$ mode in a cylindrical cavity. Thus, it is inherently $3 \mathrm{D}$, even without extraction. The beam is deflected sideways and lies in a plane containing the maximum axial electric field.

The RBTO uses an annular cavity with a ribbon-like beam fanning its way radially across the cavity, with the beam deflected up and down (along the axis of the annular cavity) uniformly around the annular cavity in the $\theta$ direction. Because the motion is uniform in $\theta$, the problem is now 2D! Because an annular cavity can support a much larger space-charge limited current than a cylindrical cavity, the beam impedance can be greatly reduced. The cavity fields for the RBTO are the TEM $_{00 \mathrm{~m}}$ mode given by

$$
\begin{aligned}
& E_{r}=(\Phi / r) \sin (m \pi z / h) \sin (\omega t-\phi), \\
& B_{\theta}=(\Phi / c r) \cos (m \pi z / h) \cos (\omega t-\phi),
\end{aligned}
$$

where $\omega=\mathrm{m} \pi \mathrm{c} / \mathrm{h}, \mathrm{h}$ is the axial length of the cavity, $\phi$ is an arbitrary phase angle, and $\Phi$ is the field amplitude. The mode number $m$ corresponds to the number of half wavelengths of the mode axially across the cavity. The closest analogue to the ABTO device is the $m=2$ mode. The unperturbed beam travels radially across the cavity with velocity $\mathrm{v}_{\mathrm{r}}$ and position $\mathrm{z}_{0}=\mathrm{h} / 2$ centered in the cavity. Note that $h$ is the only length which sets the cavity frequency for this mode.

The single-particle perturbed axial position of a beam electron is given by

$$
\mathrm{d}^{2} z / \mathrm{dt}^{2}=-\left(\mathrm{e} / \mathrm{mm}_{\mathrm{e}}\right) \mathrm{v}_{\mathrm{r}} \mathrm{B}_{\theta}
$$

where $\gamma=\left(1-\beta^{2}\right)^{-1 / 2}, \beta=v_{r} / c$, and $m_{e}$ is the electron mass. Ignoring space-charge effects, or assuming a very relativistic beam, $v_{r}$ may be treated as constant and the particle radius is $r(t)=r_{i}+\beta c t$, where $r_{i}$ is the radius of the beam at injection into the cavity. The beam travels radially across the cavity and is absorbed at radius $r_{0}=r_{i}+d$, where $d$ is the radial width of the cavity. Note, for a radially-inward directed beam, $\beta, v_{r}$, and $d$ are all negative. Because we are calculating the perturbed axial position, to lowest order in $\Phi$, we evaluate the RHS at the unperturbed position $z_{0}=h / 2$. For convenience, we define several phase angles: $\Theta \equiv \omega t ; \Theta_{d} \equiv \omega d / v_{r}$;

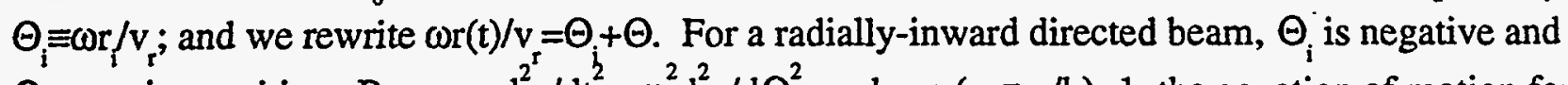
$\Theta_{\mathrm{d}}$ remains positive. Because $\mathrm{d}^{2^{2}} \mathrm{z} / \mathrm{dt} \mathrm{t}^{2}=\omega^{2} \mathrm{~d}^{2} \mathrm{z} / \mathrm{d} \Theta^{2}$, and $\cos \left(\mathrm{m} \pi \mathrm{z}_{0} / \mathrm{h}\right)=1$, the equation of motion for the beam reduces to 


$$
\begin{aligned}
& d^{2} z(\Theta) / d \Theta^{2}=-\left(e \Phi / y m_{c} \omega c\right) \cos (\Theta-\phi) /\left(\Theta_{i}+\Theta\right) \\
& \zeta(\Theta) \equiv z(\Theta)-z 0=-\left(e \Phi / y m_{e} \omega c\right) \int_{0}^{\Theta} d \Theta^{\prime} \int_{0}^{\Theta^{\prime}} d \Theta^{\prime \prime} \cos \left(\Theta^{\prime \prime}-\phi\right) /\left(\Theta_{i}+\Theta^{\prime \prime}\right) .
\end{aligned}
$$

In crossing the cavity, the particle looses energy

$$
\mathrm{m}_{\mathrm{e}} \mathrm{c}^{2} \Delta \gamma=-\mathrm{e} \int_{0}^{d / v_{r}} \mathrm{dt} \mathrm{v}_{\mathrm{r}} \mathrm{E}_{\mathrm{r}}(\mathrm{r}(\mathrm{t}), \mathrm{z}(\mathrm{t}))
$$

To lowest order in $\Phi$, linearizing $\sin (\mathrm{m} \pi \zeta / \mathrm{h})$ gives

$$
\Delta \gamma=\gamma^{-1}\left(\mathrm{e} \Phi / \mathrm{m}_{\mathrm{e}} \mathrm{c}^{2}\right)^{2} \int_{0}^{\Theta_{d}} \mathrm{~d} \Theta \int_{0}^{\Theta} \mathrm{d} \Theta^{\prime} \int_{0}^{\Theta^{\prime}} \mathrm{d} \Theta^{\prime \prime} \sin (\Theta-\phi) \cos \left(\Theta^{\prime \prime}-\phi\right) /\left(\Theta_{\mathrm{i}}+\Theta\right)\left(\Theta_{\mathrm{i}}+\Theta^{\prime \prime}\right)
$$

Averaging over initial phase angle gives

$$
\left\langle\Delta \gamma=(1 / 2 \gamma)\left(e \Phi / m_{c} c^{2}\right)^{2} \int_{0}^{\Theta_{d}} d \Theta \int_{0}^{\Theta} d \Theta^{\prime} \int_{0}^{\Theta^{\prime}} d \Theta^{\prime \prime} \sin \left(\Theta-\Theta^{\prime \prime}\right) /\left(\Theta_{i}+\Theta\right)\left(\Theta_{i}+\Theta^{\prime \prime}\right) .\right.
$$

This can be integrated to give

$$
\begin{aligned}
& \left\langle\Delta \gamma=(A / 2 \gamma)\left(e \Phi / m_{e} c^{2}\right)^{2}\right. \\
& A \equiv\left(f\left(\Theta_{i}+\Theta_{d}\right)-f\left(\Theta_{i}\right)\right) \sin \Theta_{d}+\left(g\left(\Theta_{i}+\Theta_{d}\right)+g\left(\Theta_{i}\right)\right)\left(1-\cos \Theta_{d}\right) .
\end{aligned}
$$

The functions $\mathrm{f}(\mathrm{z}) \equiv \mathrm{Ci}(\mathrm{z}) \sin (\mathrm{z})-\mathrm{si}(\mathrm{z}) \cos (\mathrm{z})$ and $\mathrm{g}(\mathrm{z}) \equiv-\mathrm{Ci}(\mathrm{z}) \cos (\mathrm{z})-\operatorname{si}(\mathrm{z}) \sin (\mathrm{z})$ are defined in terms of Sine and Cosine integrals. ${ }^{2}$

The power gained by the cavity fields due to this loss of energy by the beam particles is

$$
P=-\left\langle\Delta p I_{b} m_{e} c^{2} / e=-\left\langle\Delta p(v / c)\left(m_{e} c^{3} / e\right)^{2},\right.\right.
$$

where $I_{b}$ is the beam current and $v \equiv I_{b} / 17 k A$ is Budker's parameter. The cavity field energy is 


$$
U=\Phi^{2}(h / 4)\left|\ln \left(1+\Theta_{d} / \Theta_{i}\right)\right|
$$

The cavity interaction $Q$ is $Q_{b}=\omega U / P$, and the growth rate is $\omega / \omega=1 / 2 Q_{b}$ or

$$
\omega / \omega=(A v / m \pi \gamma) / l \ln \left(1+\Theta_{d} / \Theta_{i}\right) \mid
$$

An obvious question at this stage is how does the growth rate of the RBTO depend on the direction of the beam? Let $a$ and $b$ be the minimum and maximum radii of the cavity, respectively. Defining the positive phase angles $\Theta_{a} \equiv \omega a / v_{r} \mid$ and $\Theta_{b} \equiv \omega b / v_{r} l$, we have for a radially outward beam $\Theta_{i}+\Theta_{d}=\Theta_{b}$ and $\Theta_{i}=\Theta_{a}$, and for a radially inward beam $\Theta_{i}+\Theta_{d}=-\Theta_{a}$ and $\Theta_{i}=-\Theta_{b}$. Using the symmetry relations $f(-z)=-f(z)$ and $g(-z)=g(z)$, it is easy to show that the growth rate is independent of the beam direction if all other parameters are the same. As will be shown later, however, this does not mean that the beam direction is unimportant. There is more than the growth rate to consider.

In Fig.2, we show an example of what the growth rate looks like as a function of annular width $d=b-a$, for the case of an inward-directed beam of voltage $1 \mathrm{MVand}$ current $50 \mathrm{kA}$, in a cavity of axial length $h=15 \mathrm{~cm}$ and fixed outer radius $b=35 \mathrm{~cm}$, and mode $m=2$. The growth rate shows the expected transit-time resonances with increasing $d$, as in the $\mathrm{ABTO}^{1}$, but here the range of variation of $d$ is limited by the axis so only two growth-rate peaks are possible. Also shown are the results of several simulations using the MRC code IVORY. As with the ABTO, space-charge depression of the beam velocity shifts the transit-time resonances to smaller values of $d$ than the theory from Eq.2, which does not contain space-charge corrections. ${ }^{1}$ This effect becomes much more pronounced as the inner radius a approaches the axis, and the second growth-rate peak is almost $180^{\circ}$ out of phase with the simple theory. Nevertheless, the magnitude of the observed growth rates is in good agreement with the simple theory.

Another obvious question is which device, the ABTO or the RBTO, has the higher growth rate? One can take the limiting case of a large radius, holding fixed the beam energy and current (ie., $\gamma$ and $v$ ) and the cavity frequency and transit-time (ie., the length $h$ and the width $d$ ). The growth rate is then inversely proportional to radius. In this sense, the RBTO would have a lower growth rate than the ABTO. Fanning the beam out in the $\theta$ direction to form a radial beam crossing a large annular cavity reduces the local strength of the cavity fields and their ability to perturb the beam, and also reduces the local strength of the beam and its ability to generate cavity fields. However, the advantage of the RBTO is that one does not have to hold the beam current constant. The annular cavity has a much higher space-charge limited current, roughly proportional to radius, which makes the growth rate nearly the same if the space-charge limited 
current is injected into each device. For the ABTO, with a $2.55 \mathrm{MV}$ beam having $5 \mathrm{kA}$ of current, injected into a cylindrical cavity with length $d=14.5 \mathrm{~cm}$, radius $7.5 \mathrm{~cm}$, and frequency $2.44 \mathrm{GHz}$, simulations showed a growth rate of $0.24 / \mathrm{ns}$ or $\mathrm{Q}_{\mathrm{b}}=32 .{ }^{1}$ For the $\mathrm{RBTO}$ with a similar frequency of $2.0 \mathrm{GHz}$, the simulation in Fig. 2 with inner radius $a=r_{0}=10 \mathrm{~cm}(\mathrm{~d}=25 \mathrm{~cm})$ showed a growth rate of $0.255 / n$ or $Q_{b}=25$. Because the relative change in cavity energy per cycle is $\Delta U / U=2 \pi / Q$, roughly a quarter of the cavity energy is being regenerated each cycle! It is hard to imagine a device doing much better in this regard than the ABTO or the RBTO.

With the optimum growth rate or $Q$ nearly the same for the two devices, this makes the power and cavity energy nearly proportional to radius and the beam impedance inversely proportional to radius for the RBTO!

The same approach used by Genoni and Proctor ${ }^{3}$ to determine an upper bound for the space-charge limited current of an annular axial electron beam can be applied here for a radial electron beam. The analogue here of the annular axial beam is a pair of radial beams injected symmetrically about the cavity center at $z=h / 2$. If $z_{1}$ and $z_{2}$ are the axial starting and ending positions for one of the beams, the result can be expressed as

$$
v_{u b}=b \delta z\left(k^{2}+\alpha^{2}\right)\left(\gamma^{2 / 3}-1\right)^{3 / 2}
$$

where $\delta z=z_{2}-z_{1}$, and $\alpha$ is the solution to

$$
\mathrm{J}_{0}(\alpha b) \mathrm{N}_{0}(\alpha \mathrm{a})-\mathrm{J}_{0}(\alpha \mathrm{a}) \mathrm{N}_{0}(\alpha \mathrm{b})=0 \text {. }
$$

Here $\mathbf{k}$ is the solution to

$$
\begin{aligned}
& \left(\alpha \tanh \left(a_{3}\right) \sin \left(a_{1}\right)-k \cos \left(a_{1}\right)\right)\left(\alpha \cos \left(a_{2}\right)-k \tanh \left(a_{4}\right) \sin \left(a_{2}\right)\right)- \\
& \left(\alpha \tanh \left(a_{3}\right) \cos \left(a_{1}\right)+k \sin \left(a_{1}\right)\right)\left(\alpha \sin \left(a_{2}\right)+k \tanh \left(a_{4}\right) \cos \left(a_{2}\right)\right)=0,
\end{aligned}
$$

where $a_{1}=k z_{1}, a_{2}=k z_{2}, a_{3}=\alpha\left(z_{1}-h / 2\right)$, and $a_{4}=\alpha\left(h-z_{2}\right)$. The relevant limiting case here is a thin beam centered in the cavity, $\mathrm{z}_{1}=\mathrm{h} / 2, \mathrm{z}_{2}=\mathrm{h} / 2+\delta \mathrm{z}, \delta \mathrm{z}<\mathrm{h}$, giving

$$
v_{u b}=b \alpha \operatorname{coth}(\alpha h / 2)\left(\gamma^{2 / 3}-1\right)^{3 / 2}
$$

This gives a space-charge limited current upper bound of $110 \mathrm{kA}$ for the standard simulation parameters : $h=15 \mathrm{~cm}, a=10 \mathrm{~cm}, b=35 \mathrm{~cm}$, and $\mathrm{V}=1 \mathrm{MeV}$.

To investigate nonlinear saturation, we have performed direct numerical integration, for one beam voltage of $1.0 \mathrm{MeV}$, of the single-particle equations of motion in the electromagnetic 
fields of the TEM ${ }_{002}$ mode to determine the nonlinear energy loss $\Delta \gamma$ as the particle radially transverses the annular cavity. The numerical integrations were carried out at a series of field amplitudes $E$, and the particle energy losses $\Delta \gamma$ were averaged over particle phase $\phi$. As shown in Fig.3, for parameters corresponding to the second theory peak at $\mathrm{d}=30.5 \mathrm{~cm}$ in Fig.2, $\Delta \gamma$ initially increases quadradically with $\Phi$, in agreement with Eq.1, but eventually saturates at $\Phi_{\text {sat }}$ and then declines for $\Phi>\Phi_{\text {sat }}$. The predicted efficiency is defined as $-\Delta \gamma /(\gamma-1)$ evaluated at $\Phi_{\text {sat }}$.

Although the linear growth rate is the same for an inward-directed and an outwarddirected radial beam, the nonlinear saturation is remarkably different. The first peak in the curve of growth rate versus d, shown in Fig. 2 , occurs at $d=16.5 \mathrm{~cm}$. For an outward-directed radial beam, the efficiency is only $7 \%$ at $\Phi_{\text {sat }}=3.2 \mathrm{MV}$. However, for an inward-directed radial beam in the same cavity, the results are much better: $32 \%$ efficiency at $\Phi_{\text {sat }}=5.7 \mathrm{MV}$. This efficiency is comparable to the $35 \%$ found for the axial BTO. ${ }^{1}$ If we go to the second peak at $\mathrm{d}=30.5 \mathrm{~cm}$ in Fig.2, the efficiency of the inward-directed radial beam increases to $51 \%$ at $\Phi_{\text {sat }}=2.6 \mathrm{MV}$. This is shown in Fig.3. For the same parameters, the efficiency of the outward-directed radial beam falls to less than $1 \%$ at $\Phi_{\text {set }}=0.5 \mathrm{MV}$ !

For extraction simulations, we concentrated on the radially inward beam at the second growth rate peak. The value of $\mathrm{d}=30.5 \mathrm{~cm}$ corresponds to an inner radius of $4.5 \mathrm{~cm}$. Spacecharge effects increase the optimum inner radius to $10 \mathrm{~cm}$ for a closed cavity, corresponding to the standard parameters: $h=15 \mathrm{~cm}, r_{0}=10 \mathrm{~cm}, r_{i}=35 \mathrm{~cm}, d=15 \mathrm{~cm}, V=1 \mathrm{MeV}$, and $I_{b}=50 \mathrm{kA}$. Because of the lack of space-charge correction to the growth rate model, parameters for the extraction simulations were not optimized. The MRC codes IVORY and $\mathrm{BTEC}^{4}$ were used for the full simulations. IVORY was used for investigating a 2D method of extraction; BTEC was used for 3D extraction calculations.

The extraction method simulated consisted of an annular opening in one or both of the cavity endplates attached to an extraction vacuum coaxial transmission line. Thus, the input power coax and the output coax are concentric and sharing the middle conductor. The inner of the coaxial conductors was attached to the lower portion of the cavity, but its radius could be varied independently from the cavity lower radius. Thus, the impedance of the output coax could be varied independently. The opening gap was a second variable. The gap could also be spanned by wires or vanes to partially continue the cavity wall across the gap and to short out the DC component of the beam current and fields. The number of vanes and their angular width were third and fourth variables. Variables 2-4 could affect the resonance by altering the frequency of the desired mode. Moreover, a large extraction opening generally perturbs the cavity sufficiently that the necessary resonances for the instability are shifted or the desired mode may cease to exist. Another practical problem occurs if the desired mode is not the lowest 
frequency in the cavity; then, other lower frequency modes may grow to disrupt the beam. All of these problems were encountered during the extraction simulations.

If there were no vanes across the gap, the cavity acted as an inverse diode, with the bottom of the cavity and the inner coax conductor charging to a significant fraction of the beam energy. This affected the resonance also by lowering the speed of the electrons and raising their transit time across the cavity. It also allowed the output coax to carry off and recirculate a significant fraction of the remaining beam energy, thereby increasing overall efficiency. This configuration is 2D, and simulations were carried out with IVORY. Both cavity endplates were open and identical, and the cavity and output coax gaps were identical and equal to $2.5 \mathrm{~cm}$. The microwave output power was $1.6 \mathrm{GW}$, and the recirculated DC beam energy was $6 \mathrm{GW}$, for a total efficiency of $4 \%$. With the cavity and output coax gaps increased to $10 \mathrm{~cm}$, the microwave output power remained unchanged at $1.6 \mathrm{GW}$, but the recirculated DC beam energy increased to $26 \mathrm{GW}$, raising the overall efficiency to $7 \%$.

When vanes were included across the gap, the problem becomes 3D and the BTEC code was used. The cavity gap with vanes was placed on only one end of the cavity, with the other end closed off. The number of vanes was varied from 8 to 16 . The angular width of each vane was varied from $1 / 8$ to $3 / 4$ of the spacing between vane centers. The inner coax was placed even with the cavity bottom at $r_{0}=10 \mathrm{~cm}$. The cavity gap was varied from $d / 2$ to $d$. The optimum case was found to be 16 vanes, each $1 / 2$ closed in $\theta$, with the cavity gap extending all the way to the bottom of the cavity. The microwave output power was $5 \mathrm{GW}$, for an efficiency of $10 \%$.

The small-signal theory and computer simulations are in agreement that the RBTO is capable of producing very large microwave power on the order of $10^{10}$ watts. Moreover, it does this at a very low impedance $(<10 \Omega)$ while retaining all the other advantages of the ABTO. These characteristics open up new possible applications. Extraction simulations showed efficiencies well below theoretical predictions, so better coupling to the cavity is needed, and this will be addressed in future simulations.

We thank M. Arman for suggesting several years ago using a radial beam to lower the impedance and $T$. Genoni for providing the single-particle nonlinear saturation code and the space-charge limited currents for a radial beam. We also thank Bob Clark for performing the BTEC simulations. The work was supported by the U.S. DOE. 


\title{
Figure Captions
}

Fig.1 RBTO radially inward $1 \mathrm{MV}, 50 \mathrm{kA}$, beam at $30 \mathrm{~ns}$. $2 \mathrm{Ghz}$ cavity with length $\mathrm{h}=15 \mathrm{~cm}$, outer radius $b=35 \mathrm{~cm}$, inner radius $a=10 \mathrm{~cm}$, annular width $d=b-a=25 \mathrm{~cm}$, mode $m=2$.

Fig. 2 Growth rate versus d, holding $b$ fixed at $35 \mathrm{~cm}$ as in Fig.1, and varying a.

Fig.3 Particle energy loss versus field amplitude for second theory peak at $\mathrm{d}=30.5 \mathrm{~cm}$ in Fig. 2 .

\author{
'Thomas J. T. Kwan, Michael A. Mostrom, and Brendan B. Godfrey, PRL 66, 3221 (1991). \\ ${ }^{2}$ Abromowitz, Milton and Stegun, Irene A. , Handbook of Mathematical Functions, National Bureau of Standards \\ Applied Mathematics Series 55, 232 (1972). \\ ${ }^{3}$ T. C. Genoni and W. A. Proctor, J. Plasma Physics 23, 129 (1980). \\ 'BTEC, maintained by Robert Clark at MRC, Albuquerque, is a derivative of the MRC code SOS.
}

\section{DISCLAIMER}

This report was prepared as an account of work sponsored by an agency of the United States Government. Neither the United States Government nor any agency thereof, nor any of their employees, makes any warranty, express or implied, or assumes any legal liability or responsibility for the accuracy, completeness, or usefulness of any information, apparatus, product, or process disclosed, or represents that its use would not infringe privately owned rights. Reference herein to any specific commercial product, process, or service by trade name, trademark, manufacturer, or otherwise does not necessarily constitute or imply its endorsement, recommendation, or favoring by the United States Government or any agency thereof. The views and opinions of authors expressed berein do not necessarily state or reflect those of the United States Government or any agency thereof. 


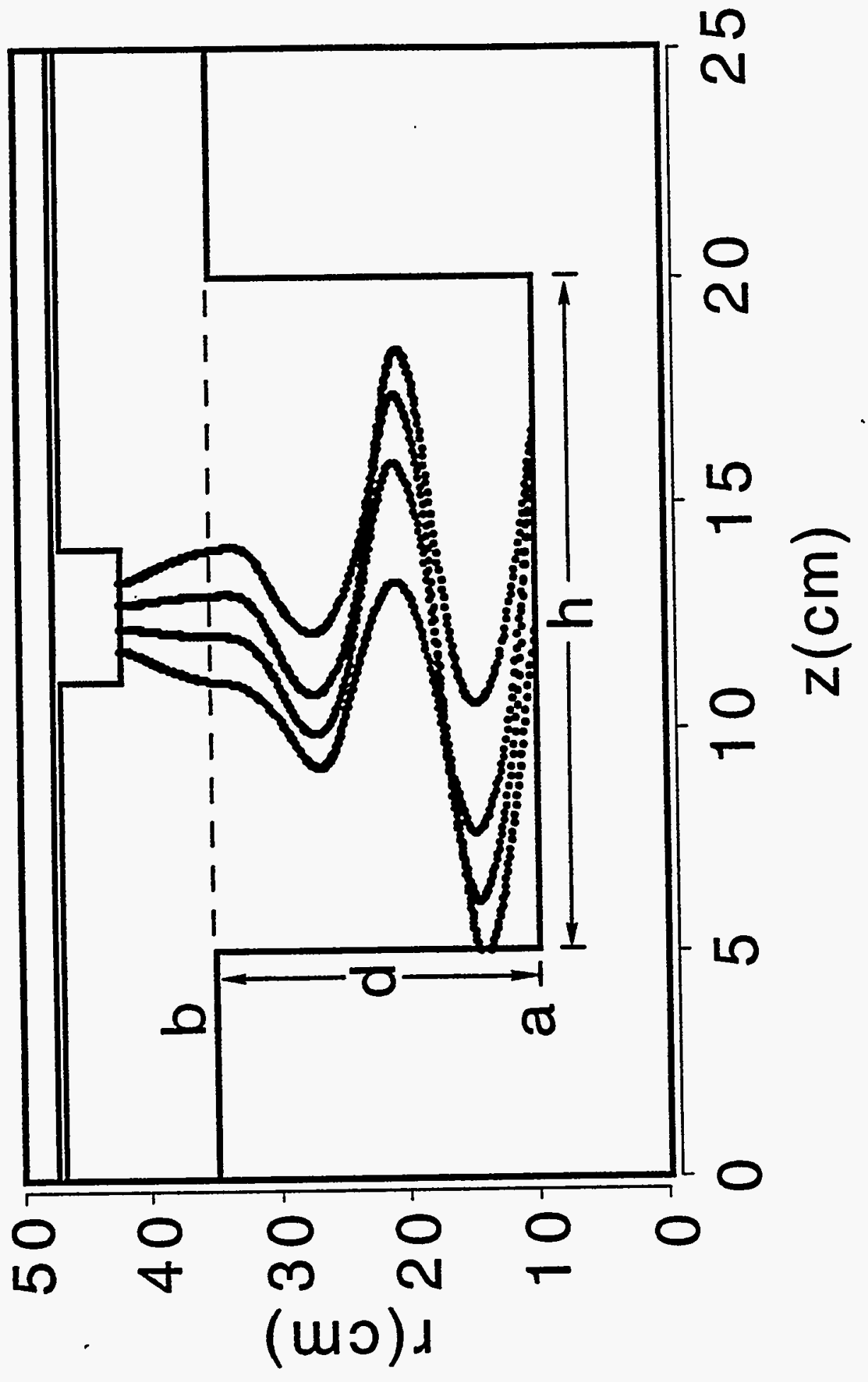




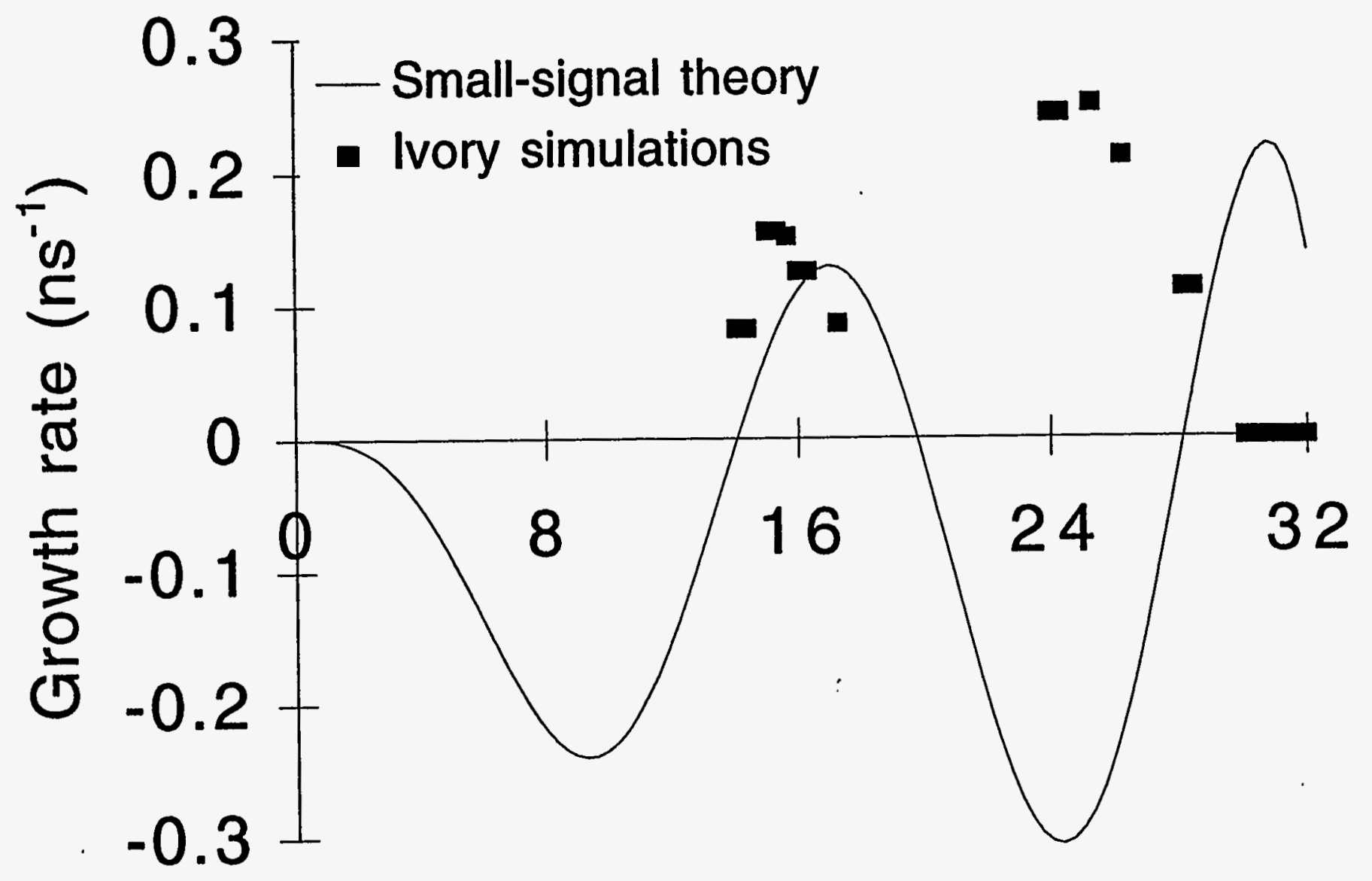

Annular cavity width $d(\mathrm{~cm})$ 


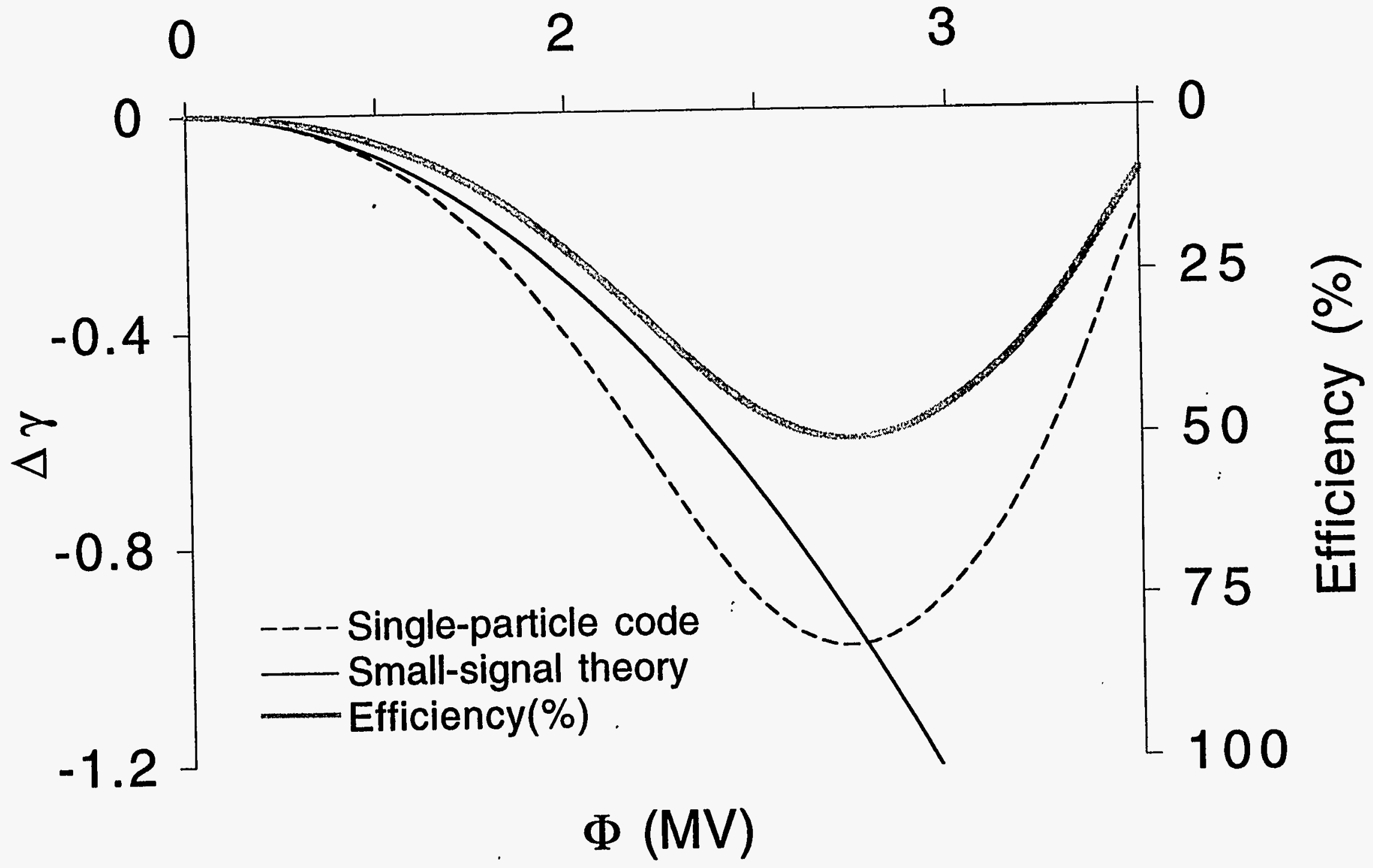

